\title{
On Tolerance Problem of Contacting Polyhedral Objects
}

\author{
Wen-Hua Pan ${ }^{1,2}$, Jing-Sin Liu ${ }^{1}$, Member, IEEE \\ ${ }^{1}$ Institute of Information Science \\ Academia Sinica \\ Nankang, Taipei 11529, Taiwan \\ ${ }^{2}$ Department of Engineering Science and Ocean Engineering \\ National Taiwan University
}

liu@iis.sinica.edu.tw

\begin{abstract}
This paper first presents the decision curve as an economical way that describes the correlations between scaling pairs $\left(\rho_{1}, \rho_{2}\right)$, small relative motion conditions (type, direction and amount) and contact configurations (feature) of two scaled convex polyhedral objects where $\rho_{i}$ denotes the scaling factor of

polyhedral object $i=1,2$. Via Analytical Equations of contact feature, the new decision curves after an intended relative motion of any type within its coherent range could be immediately obtained by taking advantage of original decision curve. We illustrate via two examples this method allows users to systematically and efficiently search a lot of design of fine motions to reorient two nominally contacting convex or nonconvex polyhedral objects with assigned tolerance of scaling factors that satisfy the requirements.
\end{abstract}

Index Terms - Tolerance, Scaling, Relative motion, Collision detection.

\section{INTRODUCTION}

Collision detection of two objects is the process of finding the geometrical or physical contact conditions between the objects which maybe moving or deformable. There is a lot of applications for this problem, like robot control and path planning, manufacturing/assembly, CAD/CAM, interactive 3D virtual environments of TV/PC games, physically-based simulation and animation, computer graphics and movie industry [1], [2]. A basic collision/contact detection problem is that of two convex Polyhedra [1]-[5], [9], [12],[17]. There are three possible contacts: Point, Line and Face contact. Hence, two objects will have seven collision configurations, four configurations (Face-Vertex, two Edges cross each other, Edge-Vertex and Vertex-Vertex) for point contact, two (two Edges touch each other and Face-Edge) for line contact and only one (Face-Face) for face contact [15],[11].

To deal with collision detection of convex polyhedral objects undergoing uniform scaling transformation (with respect to an interior point called seed point) [7], [1] and relative motion, it is worth noting that a continuum, rather than a discrete set, of scaled contact configurations exist. We developed numerical collision detection method based on inner-outer ellipsoids [13] and decision curve [6],[10],[13] which we call Fast Collision Detection Method (FCDM) detailed in Fig. 1. FCDM, which is based on a few known contact scaling pairs combined with Analytical Equations of contact features, could found out the contact configurations and a continuum of contact scaling pairs. This method could efficiently establish the correlations between the scaling pairs, contact configurations and relative fine motion conditions using much less computation time than (enhanced) GJK algorithm [8], [12], [17], provided that the original decision curve is available a priori (Fig.2) [10], [13]. By contrast, the conventional collision detection methods, like GJK, BVH, Ray-Triangle, can treat one relative configuration after an assigned relative motion per run. In addition, GJK suffers from the ill-conditioned numerical problem that may occur in applying GJK to two convex polyhedral objects whose size is significantly different ([1] p.143), and thus can't be automated. Those algorithms only could confirm the contact configuration for one scaling pair at one run, not the whole continuum as our method. This prohibits their usefulness in industrial design problems, which would essentially require a number of trial runs. We have demonstrated that this method is an efficient design tool for the assembly problem [10]. FCDM could be applied to different design problems where size and contact of convex polyhedral objects is the main concern in a simple and flexible way. Building on our previous work on FCDM [6], [10], [13], this paper further extends the use of FCDM in tolerance problem, assuming that the possible range of variation of size/dimension of object is assigned.

The paper is organized as follows. Section 2 reviews Fast Collision Detection Method (FCDM) for construction of decision curve for scaled convex polyhedral objects with or without relative motion detailed in the flowcharts of Fig. 1 and Fig. 2 that could efficiently find the correlation among scaling pairs, contact configurations (feature) and relative motion conditions (type, direction and amount). Section 3 contains the decision curves of two illustrated examples to be used in Section 4 . Section 4 is a case study of the tolerance problem for the two examples. Finally, conclusion is made in Section 5.

\section{DECISION CURVES WITH OR WITHOUT RELATIVE MOTION}

Decision curve for a pair of scaled convex polyhedral objects is defined as a graphical description of a continuum of scaling pairs which could make the two scaled polyhedral object just contact at one point. It is a piecewise continuous curve built by piecing together many different line segments in the $\rho 1-\rho 2$ plane of scaling factors, where $\rho_{i}$ denotes the scaling factor of object $i=1,2$. Each line segment graphically describes a continuum of scaling pairs which make the two objects just contact at one point with the same contact feature.

The decision curve separates the plane of scaling factors into two subregions: overlap and non-contact. Geometrically, the scaling pairs (configurations) above this curve means the two objects are overlapping, while those below the curve are separating. The scaling pair on the curve are contact scaling pairs which the convex polyhedral objects contact at one 


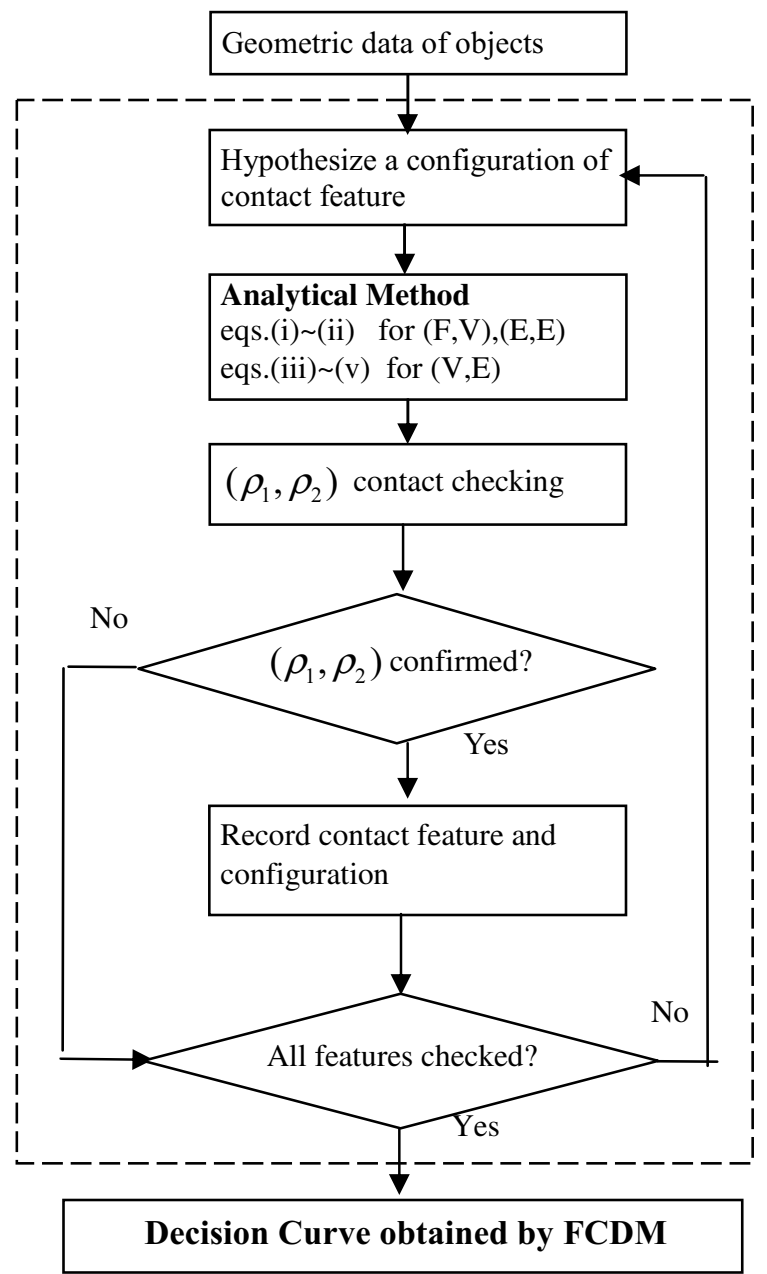

Fig. 1. Flowchart of Fast collision detection method (FCDM)

single point. This section will briefly recall the analytical method used in the flowcharts of Fig.1 and Fig.2 for searching a variety of correlated combinations of contact scaling pairs, relative motion and contact feature satisfying the requirements.

\section{A. Analytical Equations Of Contact Feature}

Given the contact status of two convex polyhedral objects, we could define its relation equation between $\rho_{1}$ and $\rho_{2}$ by the geometric data of two objects [6]. In this paper, we consider about three non-degenerate, elementary point contact statuses: face-vertex $(\mathrm{F}, \mathrm{V})$, edge-edge cross $(\mathrm{E}, \mathrm{E}) \mathrm{c}$ and edge vertex $(\mathrm{E}, \mathrm{V})$, since these statuses are the most frequently occurring than others. However, the method is equally applicable to other contact features. The Analytical Equations[6], [10], [13] of three basic contact statuses could be expressed by five set equations listed in Appendix: either a linear equation (i)-(ii) that describe a continuum of scaled contact configurations with a specific contact feature of $(\mathrm{F}, \mathrm{V})$ contact or (E,E) contact, or a single contact scaling pair of $(\mathrm{E}, \mathrm{V})$ (iii)-(v) that describes the instantaneous transition between two contact features (the intersection of two lines), i.e. instantaneous transition between $(\mathrm{F}, \mathrm{V}) \&(\mathrm{E}, \mathrm{E})$,

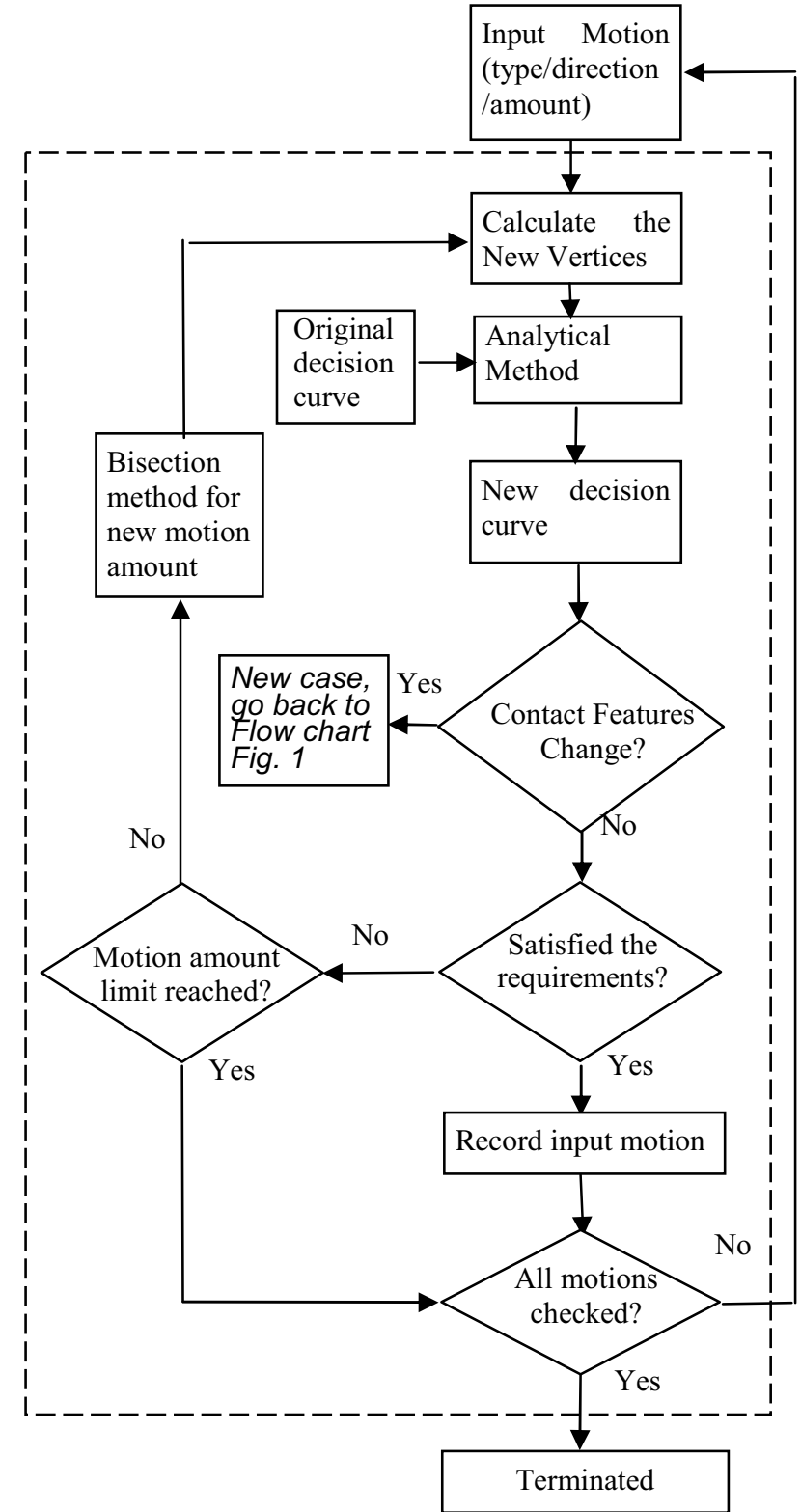

Fig. 2 Flowchart of FCDM for incrementally building the family of new decision curves after relative motion

$(\mathrm{E}, \mathrm{E}) \&(\mathrm{E}, \mathrm{E})$, or $(\mathrm{F}, \mathrm{V}) \&(\mathrm{~F}, \mathrm{~V})$. With these equations at hand, as shown in Fig. 1, we could efficiently to calculate a contact scaling pair $\left(\rho_{1}, \rho_{2}\right)$ corresponding to a specific contact feature, e.g. $(\mathrm{F}, \mathrm{V}),(\mathrm{E}, \mathrm{E}),(\mathrm{V}, \mathrm{E})$, given the vertices of this feature. However, this step could not verify whether this contact status exists in the real convex polyhedral objects because the real objects are solid while Analytical Equations only considers about vertices, edges and faces. It's possible that two objects already be overlapped before this feature contact. Secondly, for the step " $\left(\rho_{1}, \rho_{2}\right)$ contact checked" in Fig. 1 many different techniques for overlap checking of convex polyhedra may be used. Repeating this process of Fig. 1 for different hypothesized contact feature, the decision curve 

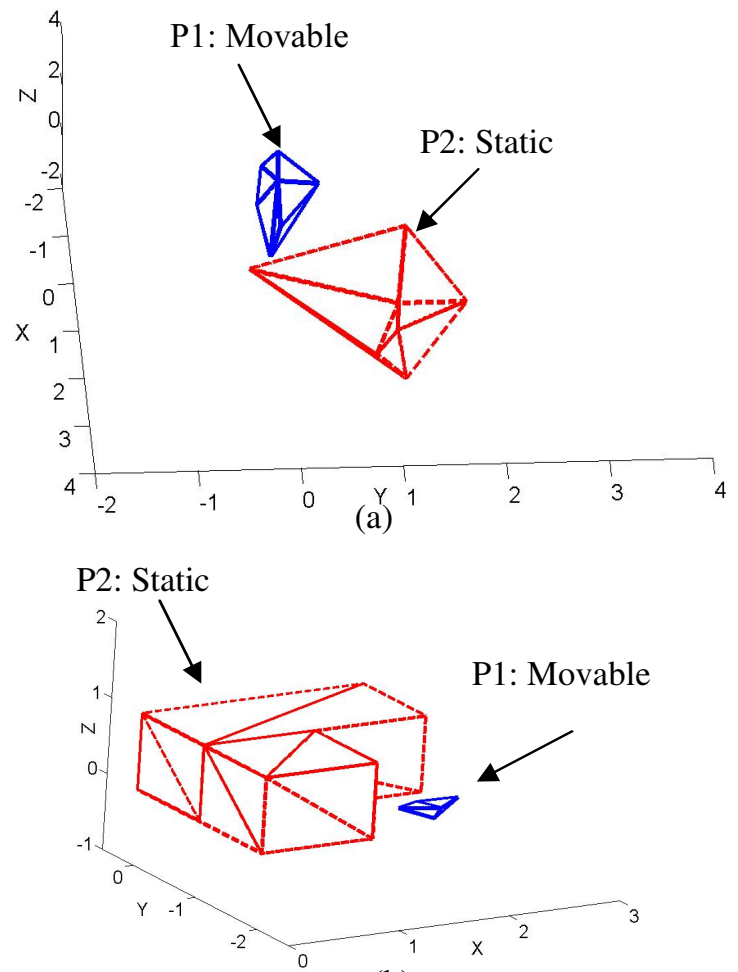

(b)

Fig. 3 (a). Example I, (b) Example II Solid, Movable object-- Polyhedron 1; Dash, Static object-- Polyhedron 2

(a continuum of contact scaling pairs) is built for two static scaled convex polyhedra.

\section{B. Procedure For New Decision Curve After Relative Motion}

Assume one object (e.g. obstacle) is static and another (robot) is movable. If the original decision curve is available, we could immediately obtain the new decision curve after arbitrary relative motion within its coherent range of motion [13] by analytical method based on new vertices of scaled objects after relative motion [10]. The process is detailed in Fig. 2. After a specified relative motion, the new vertices of movable object could be calculated through the geometric relations [10]. Analytical Equations of contact features for the new contact configuration of the same feature as original decision curve could be updated numerically by substituting the new vertices of movable object calculated through the geometric relations [10]. The updated equations are then employed to build the new decision curve after motion, which is analytically defined by updated equations, not by discrete data set of a large number of contact scaling pairs. In this way, a family of new decision curves after motion, which are very similar to the original one [10], [13], are built directly by following the procedure depicted in flowchart Fig.2. These curves are useful to detect the contact status of two scaled polyhedral objects in different moving situations.

The statement "Satisfied the requirements" in Fig. 2 offers a flexibility of incorporation of requirements tailored to different applications in a quite simple way. Taking advantage

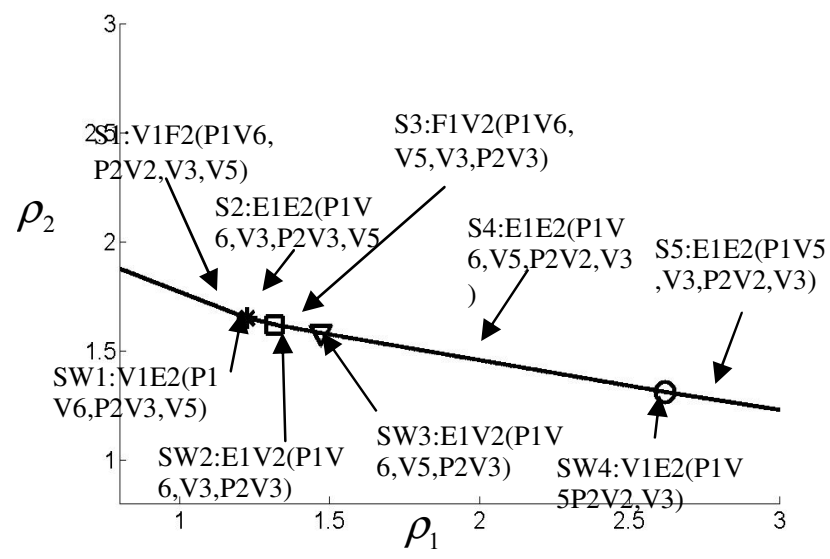

(a)

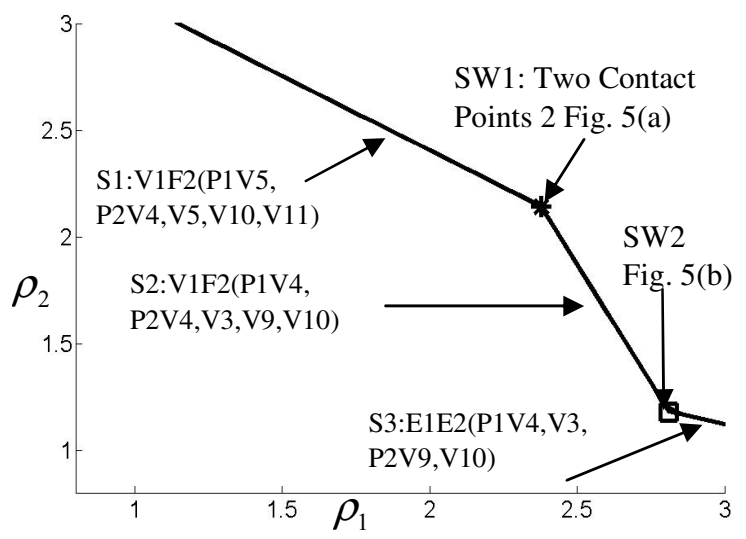

(b)

Fig. 4.Decision curves of Fig. 3 (a), (b).

of original decision curve, this procedure allows an efficient implementation to systematically examine a lot of possible solutions based on a family of new decision curves constructed for a variety of situations to the specific design problem concerning scaling and relative motion (e.g. assembly [10], range of motion [13]) in very short computation time. This will be further illustrated in this paper by applying to tolerance problem. Note that the determination "Contact Features Changed" in Fig. 2 is to verify whether there is any existing contact feature vanished/merged or there is any newly appeared contact feature for the input relative motion amount. This checking is based on that as the relative motion amount is beyond its coherent range, the two scaled polyhedral objects have different contact feature after motion [13].

\section{TWO NUMERICAL EXAMPLES FOR CASE STUDY}

Fig. 3 shows the 3D image of the two examples used for demonstration, where each example has a pair of polyhedral objects initially separated. Example 1 consists of two convex polyhedral objects, and Example 2 consists of one convex polyhedral object, and another is non-convex polyhedral object, which could be decomposed into a few convex polyhedral objects. The seed point for scaling is the center of

each object. For each example, Polyhedron 1 (solid line in the figure) is movable, and Polyhedron 2 (dash line in the figure) 

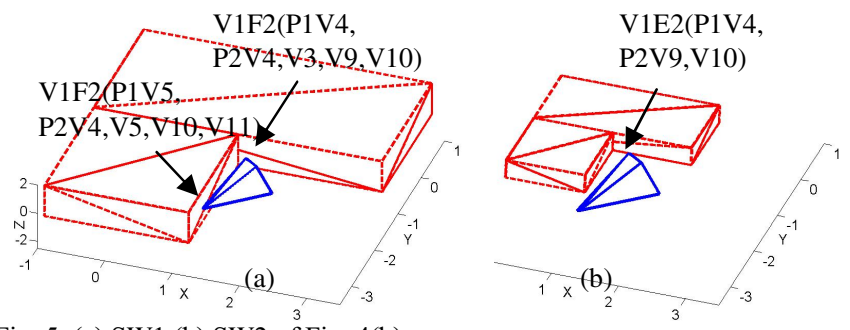

Fig. 5 (a) SW1 (b) SW2 of Fig. 4(b)

(a) two contact points :V1F2(P1V5, P2V4, V5, V10, V11) \& V1F2(P1V4, P2V4,V3,V9,V10) at scaling pair $(2.3780,2.1409)$

(b) one contact point : V1E2(P1V4, P2V9,V10) at scaling pair $(2.8110,1.1795)$

is static. In this study, the allowable scaling range is set as $\left(\rho_{1}, \rho_{2}\right) \in[0.8,3]^{2}$ in both examples. The decision curve constructed for each example via Fig. 1 is shown in Fig. 4. The contact feature on each line segment or a switching point is also indicated on Fig 4(a), (b).

The symbol on the decision curve in Fig. 4, for example $\mathrm{S} 1: \mathrm{V} 1 \mathrm{~F} 2(\mathrm{P} 1 \mathrm{~V} 6, \mathrm{P} 2 \mathrm{~V} 2, \mathrm{~V} 3, \mathrm{~V} 5)$ means the segment 1, combined by the Vertex which is the $6^{\text {th }}$ of Polyhedron 1 , and the face that defined by $2^{\text {nd }}, 3^{\text {rd }}$ and $5^{\text {th }}$ vertices of Polyhedron 2.For Example I, there are 5 segments $(\mathrm{S} 1 \sim \mathrm{S} 5)$, and 4 switching points $(\mathrm{SW} 1 \sim \mathrm{SW} 4)$ in $\left(\left(\rho_{1}, \rho_{2}\right) \in[0.8,3]^{2}\right)$. For Example II, there are 3 segments $(\mathrm{S} 1 \sim \mathrm{S} 3)$, and 2 switching points (SW1, $\mathrm{SW} 2)$ in $\left(\left(\rho_{1}, \rho_{2}\right) \in[0.8,3]^{2}\right)$. However, is very special: there are two contact points at SW1: V1F2(P1V5, $\mathrm{P} 2 \mathrm{~V} 4, \mathrm{~V} 5, \mathrm{~V} 10, \mathrm{~V} 11)$ and V1F2(P1V4, P2V4,V3,V9,V10). This reveals a very important distinguishing character between nonconvex and convex polyhedral objects contact. For convex polyhedral object, there is a unique contact point corresponding to a contact configuration on the decision curve characterizing a continuum of contact configurations. For nonconvex polyhedral object, it's possible to make contact without overlapping at more than one point at the same scaling pair. This situation could be recognized very clearly from the decision curve. Based on our method, the contact feature of each segment is known. Hence, comparing the contact features of two line segments linking by the switching point, we could know which kind of contact points is this switching point.

More specifically, SW1 of Fig. 4(b) is between the line segment V1F2(P1V5, P2V4,V5,V10,V11) and the line segment V1F2(P1V4, P2V4,V3,V9,V10). The contact points of those two segments could not be merged together, because there are no common features of those configurations. Thus, SW1 is the switching point which has two contact points(Fig. 5(b)). About SW2 of Fig. 4(b), the line segments V1F2(P1V4, P2V4,V3,V9,V10) and E1E2(P1V4, V3, P2V9,V10) have the common features. The contact points could be merged at SW2 as V1E2(P1V4, P2V9, V10) (Fig. 5(b)).

\section{TOLERANCE PROBLEM}

In the real world, manufacturing always produce products with tolerance. Scaling tolerance is common in manufacturing process, e.g. molding, forging... etc. It defines the possible range of variation of size/dimension of a nominal design of a product. This section demonstrates via the two examples in Fig. 3 the use of decision curves for the tolerance problem in manufacturing. We consider tolerance of scaling, expressed in terms of nominal scaling factor \pm tolerance. That is, the shape of each object is unchanged and only its size and thus relative configuration is slightly changed by the a priori assigned tolerance in which we assume that there is a possible range of variation of object size. Our aim is to design the suitable relative motion conditions to reorient two nominally contacting objects whose size has been changed a little for precise assembly. This tolerance problem, if solvable, may have infinitely many solutions. Using new decision curves after motions, one could rapidly confirm if a range of motion conditions fits the design requirement.

We assume the scaling of both objects in contact in Example 1 and Example 2 has a tolerance $\pm 2 \%$. The requirement is to make the two objects whose size is rescaled by their respective assigned tolerance still in contact; our aim is to find out the suitable fine motion conditions to reorient/rearrange the static and movable objects whose size is deviated from the nominal size during the assembly process.

Following the flowchart on Fig. 2, the search requirements for scaling tolerance problem could be written as:

$$
\begin{aligned}
& A:\left(\rho_{1, \text { new }} \geq 1.02 \rho_{1, \text { old }}\right) \&\left(\rho_{2, \text { new }} \geq 1.02 \rho_{2, \text { old }}\right) \\
& B:\left(\rho_{1, \text { new }} \geq 1.02 \rho_{1, \text { old }}\right) \&\left(\rho_{2, \text { new }} \leq 0.98 \rho_{2, \text { old }}\right) \\
& C:\left(\rho_{1, \text { new }} \leq 0.98 \rho_{1, \text { old }}\right) \&\left(\rho_{2, \text { new }} \geq 1.02 \rho_{2, \text { old }}\right) \\
& D:\left(\rho_{1, \text { new }} \leq 0.98 \rho_{1, \text { old }}\right) \&\left(\rho_{2, \text { new }} \leq 0.98 \rho_{2, \text { old }}\right)
\end{aligned}
$$

For each of the above requirements, we try to find out the motion conditions that could satisfy it and obtain the motion ranges for precise assembly when the scaling pairs have tolerance. We could choose any scaling pairs on the original decision curve, which means the two objects are in contact, and if the scaling process has some tolerance, we want to search the coherent relative motions that could make the two objects still in contact.

In Example 1, we assume the initial design of scaling pair is that two objects contact at SW3, but the scaling pair has a tolerance $\pm 2 \%$ because manufacturing. The scaling tolerance range of SW3 is shown as the shadow range in Fig. 6. Of course, there are many possible relative motion conditions that could satisfy this requirement, here we only illustrate two types of relative motion: (i)Screw along the line linking two seed points, and (ii) a Translation along $\mathrm{Z}$ axis followed by a Rotation around $Z$ axis. Given the requirements of scaling tolerance, and the searching range of relative motion amount, then the new four decision curves passing each new scaling pair of SW3 are computed via automated process of Fig. 2.

Examining the new decision curves after relative motion, it can be seen that the minimum motion amount is the amount that makes the new SW3 outside the shadow area in Fig. 6, and as close to the boundary of shadow as possible. Therefore, four alternative motions are found feasible to reorient the resized objects to keep the same contact at the new SW3 for four combinations of scaling tolerance. In this case, the 
assembly process must be modified via relative motion found as listed on Table 1.

Following the same procedure, we could check the tolerance condition for Example 2 at scaling pair on SW1, where the intended relative motions are: (i)a Translation along $\mathrm{Y}$ axis, and (ii)a Translation along $\mathrm{Y}$ axis followed by a Rotation around $Z$ axis. The result is shown in Table 2 and Fig. 7. The scaling tolerance problem of non-convex polyhedral object also could be solved by this method.

TABLE 1. Example 1. Assigned a scaling tolerance $\pm 2 \%$ (at SW3) to find coherent relative motion conditions (see also Fig. 5). TZ: translation along $Z$ axis, $R Z$ : rotation around $Z$ axis

\begin{tabular}{|l|l|l|}
\hline$\rho_{1}$ & \multicolumn{1}{|c|}{$+2 \%$} & \multicolumn{1}{|c|}{$-2 \%$} \\
\hline$+2 \%$ & $\begin{array}{l}\text { Sequirement A } \\
\text { Screw : translation - } \\
0.0054 \text { and rotation - } \\
0.0054^{\circ} \text { Fig. 6(2) }\end{array}$ & $\begin{array}{l}\text { Requirement C }-0.1178+\mathrm{RZ}-6^{\circ} \\
\text { Fig.6 (4) }\end{array}$ \\
\hline$-2 \%$ & $\begin{array}{l}\text { Requirement B } \\
\text { TZ 0.25+ RZ 7.0191 }\end{array}$ \\
& Fig.6 (3) & $\begin{array}{l}\text { Requirement D } \\
\text { Screw : translation } \\
0.0051 \text { and rotation }\end{array}$ \\
& & $0.0051^{\circ}$ Fig.6 (5) \\
\hline
\end{tabular}

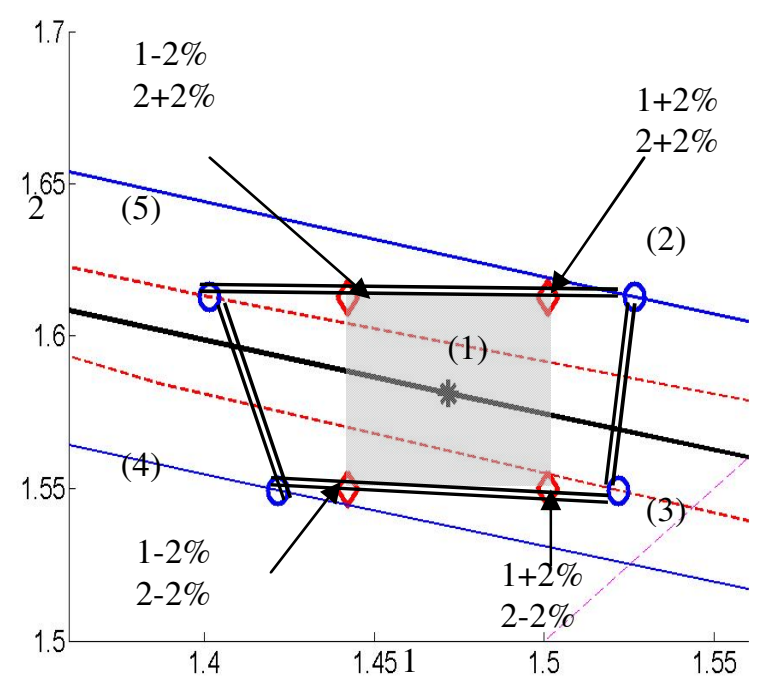

Fig. 6 Example 1. *: Original SW3; $\diamond$ : scaling tolerance SW3; O: new SW3 after motion

(1) Given the original decision curve of nominal design ;

new decision curve after motion: (2) Screw motion composed of a translation -0.0054 and a rotation $-0.0054^{\circ}$; (3) TZ $0.25+\mathrm{RZ} 7.0191^{\circ}$; (4) $\mathrm{TZ}-0.1178+\mathrm{RZ}-6^{\circ}$; (5) Screw motion composed of a translation 0.0051 and a rotation $0.0051^{\circ}$;

Shadow area: scaling tolerance range;

Double line area: computed coherent motion range
TABLE 2. Example 2. Assigned a scaling Tolerance $\pm 2 \%$ (at SW1) to find coherent relative motion conditions (see also Fig.7) $\mathrm{TY}$ : translation along $\mathrm{Y}$ axis, $\mathrm{RZ}$ : rotation around $\mathrm{Z}$ axis

\begin{tabular}{|l|l|l|}
\hline$\rho_{1}$ & \multicolumn{1}{|c|}{$+2 \%$} & \multicolumn{1}{c|}{$-2 \%$} \\
$+2 \%$ & $\begin{array}{l}\text { Requirement A } \\
\text { TY }-0.0337+\text { RZ 2 } \\
\text { Fig.7 (2) }\end{array}$ & $\begin{array}{l}\text { Requirement C } \\
\text { TY 0.0193 } \\
\text { Fig.7(4) }\end{array}$ \\
\hline$-2 \%$ & $\begin{array}{l}\text { TY -0.0193 } \\
\text { Requirement B } \\
\text { Fig. 7 (3) }\end{array}$ & $\begin{array}{l}\text { Requirement D } \\
\text { TY 0.0313 + RZ-2 } \\
\text { Fig. 7 (5) }\end{array}$ \\
\hline
\end{tabular}

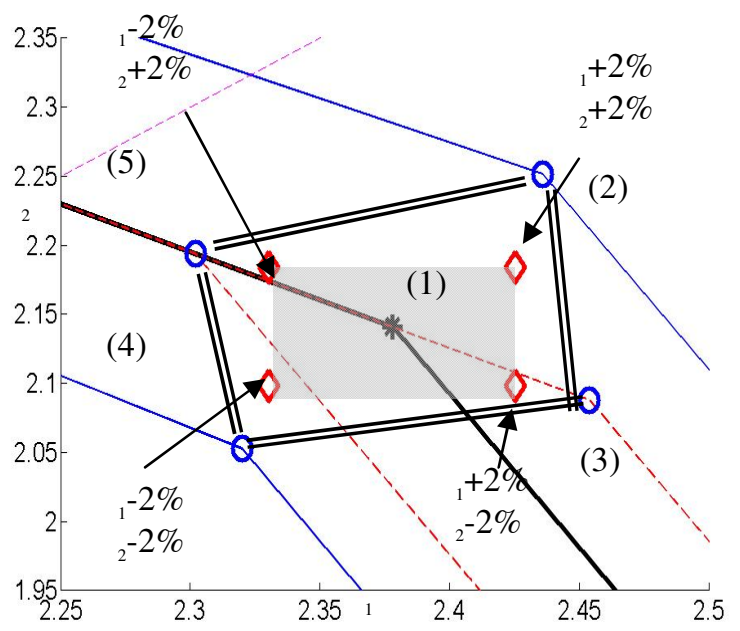

Fig. 7. Example 2. *: original SW1; $\diamond$ : scaling tolerance at SW1; O: new SW1 after motion

(1) Given the original decision curve of nominal design; new decision cureve after motion: (2) TY $-0.0337+\mathrm{RZ} 2^{\circ}$; (3) TY -0.0193 ; (4) TY 0.0193 ;

(5) TY $0.0313+\mathrm{RZ}-2^{\circ}$;

Shadow area: scaling tolerance range;

Double line area : computed coherent motion range 


\section{CONCLUSION}

In this paper, we demonstrate via two examples the practicality of Fast Collision Detection Method (FCDM), which could efficiently establish an appropriate correlation for contact scaling pairs associated with contact feature and motion amount of an intended relative motion of any type, as a flexible and useful design tool for tolerance problem of two contacting convex or non-convex polyhedral objects. Within the assigned range of tolerance of scaling pairs, FCDM could systematically and automatically search the corresponding contact statuses and relative motion conditions needed to reorient the rescaled objects to contact satisfying the requirements. Therefore it is useful to support the designer's decision of choosing an appropriate combination meeting the requirements.

\section{ACKNOWLEDGMENT.}

The authors appreciate Prof. Masatomo Inui, Ibaraki University, Japan for helpful discussions. This work was supported by National Science Council of R.O.C. under contract NSC 96-2221-E-001-018-MY2.

\section{REFERENCES}

[1] Gino van der Bergen, Collision detection in interactive $3 D$ environments, Morgan Kaufmann Publishers, 2004.

[2] C. Ericson, Real-time collision detection, Elsevier, 2005.

[3] P. Jimenez, F. Thomas and C. Torras, "Collision detection algorithms for motion planning," in J.-P. Laumond (Ed.): Robot motion planning and control, LNCIS 229, Ch6, 1998.

[4] C. Fares and Y. Hamam, "Collision detection for rigid bodies: a state of the art review," 15th Int. Conf. Computer Graphics and Applications (GraphiCon'2005), Russia, June 2005.

[5] P. Jimenez, F. Thomas, C. Torras, "3D collision detection: a survey," Computer \& Graphics, vol.25, no. 2, pp.269-285, 2001.

[6] J.-S. Liu, Y.H. Tsao, W.-Y. Ku, W.-H. Pan and Y.-Z. Cheng, "Exact Collision Detection for Scaled Convex polyhedral Objects," Technical report TR 2007-03, Institute of Information Science, Academia Sinica, Jan. 2007.

[7] C.J. Ong and E.G. Gilbert, "Growth distances: new measures for object separation and penetration," IEEE Trans. on Robotics and Automation, vol.12, no. 6, pp.888-903, 1996.

[8] C.J. Ong and E.G. Gilbert, "Fast versions of the Gilbert-Johnson-Keerthi distance algorithm: additional results and comparisons," IEEE Trans. on Robotics and Automation, vol. 4, pp. 531-539, 2001.

[9] F. Yang and M. M. Marefat, "Achieving Desired Contact State Transitions of Polyhedral Parts with Compliant Motions," 2005 IEEE International Conference on Robotics and Automation, pp.4206-4211, 2005.

[10] W.-H. Pan, J.-S. Liu and W.-Y. Ku, "Fast collision detection method for the scaled convex polyhedral objects with relative motion," IEEE Int. Symp. Assembly and Manufacturing, , pp.184-190, Ann Arbor, Michigan, 2007.

[11] L. Luo and M. Nahon, "A compliant contact model including interference geometry for polyhedral objects," ASME Trans. of Computational and Nonlinear Dynamics, vol. 1, pp.150-159, 2006.

[12] E.G. Gilbert, D. W. Johnson, and S.S. Keerthi, "A fast procedure for computing the distance between complex objects in three-dimensional space," IEEE Trans. Robot Automation, vol. 4, pp. 193-203, Apr. 1988.

[13] J.-S. Liu, W.-H. Pan, "Automatic computation of coherent range of motion for scaled convex polyhedra," IEEE Robio2007, Sanya, Hainan, China, Dec. 2007

[14] J.-S. Liu, J.-.I. Kao and Y.-Z. Chang ,"Collision detection of deformable polyhedral objects via inner-outer ellipsoids," IEEE/RSJ Int. Conf. Intelligent Robots and Systems, pp.5600-5605, Beijing, China, 2006.
[15] J. Xiao and X. Ji, "Automatic generation of high-level contact space," The International Journal of Robotics Research, vol.20, no.7, pp.584606, 2001.

[16] L.-W. Tsai, Robot Analysis, 1999, John Wiley\& Sons, Inc.

[17] S. Cameron, "Enhancing GJK: computing minimum and penetration distances between convex polyhedra," 1997 IEEE International Conference on Robotics and Automation, vol 4, pp. 3112-3117, 1997

\section{APPENDIX.}

List of contact equations of $(\mathrm{V}, \mathrm{F}),(\mathrm{V}, \mathrm{E}),(\mathrm{E}, \mathrm{E})$ contact feature in the plane of scaling factors

Notation

$s_{i}:$ the seed point of polyhedron $\mathrm{i}, \mathrm{i}=1,2$

$v_{i j}$ : the jth vertex of polyhedron $\mathrm{i}, \mathrm{i}=1,2$

$c_{1}$ is the contact point

$\vec{n}_{1}=$ the unit normal vector of contact face of polyhedron 1

$\operatorname{det}[V S] \equiv \operatorname{det}\left[-\left(v_{12}-v_{11}\right),\left(s_{1}-s_{2}\right),\left(v_{22}-v_{21}\right)\right]$

$\operatorname{det}[V V] \equiv \operatorname{det}\left[-\left(v_{12}-v_{11}\right),\left(v_{11}-s_{1}\right),\left(v_{22}-v_{21}\right)\right]$

$\operatorname{det}[A] \equiv \operatorname{det}\left[-\left(v_{12}-v_{11}\right),\left(v_{21}-s_{2}\right),\left(v_{22}-v_{21}\right)\right]$

$\operatorname{dot}($ nas $) \equiv \operatorname{dot}\left(\vec{n}_{1},\left(a_{1}-s_{1}\right)\right)$

$\operatorname{dot}(n s s) \equiv \operatorname{dot}\left(\vec{n}_{1},\left(s_{1}-s_{2}\right)\right)$

$\operatorname{dot}(n c s) \equiv \operatorname{dot}\left(\vec{n}_{1},\left(c_{2}-s_{2}\right)\right)$

The linear equations of contact are listed below.

(i) $(\mathrm{F}, \mathrm{V})$ contact

$\rho_{2}=\frac{\rho_{1} \operatorname{dot}\left(\vec{n}_{1},\left(v_{21}-s_{1}\right)\right)+\operatorname{dot}\left(\vec{n}_{1},\left(s_{1}-s_{2}\right)\right)}{\operatorname{dot}\left(\vec{n}_{1},\left(c_{2}-s_{2}\right)\right)}$

(ii) $(\mathrm{E}, \mathrm{E})$ contact

$\rho_{2}=\frac{\operatorname{det}\left[\left(v_{12}-v_{11}\right), s_{1}-s_{2}+\rho_{1}\left(v_{11}-s_{1}\right), v_{22}-v_{21}\right]}{\operatorname{det}\left[\left(v_{12}-v_{11}\right), v_{21}-s_{2}, v_{22}-v_{21}\right]}$

(iii) $(\mathrm{E}, \mathrm{V})$ contact as an instantaneous transition between $(\mathrm{F}, \mathrm{V})$ contact and $(\mathrm{E}, \mathrm{E})$ contact

$$
\begin{aligned}
& \rho_{1}=\frac{\operatorname{dot}\left(\vec{n}_{1},\left(c_{2}-s_{2}\right)\right) \times \operatorname{det}[V S]-\operatorname{dot}\left(\vec{n}_{1},\left(s_{1}-s_{2}\right)\right) \times \operatorname{det}[A]}{\operatorname{dot}\left(\vec{n}_{1},\left(v_{11}-s_{1}\right)\right) \times \operatorname{det}[A]-\operatorname{dot}\left(\vec{n}_{1},\left(c_{2}-s_{2}\right)\right) \times \operatorname{det}[V V]} \\
& \rho_{2}=\frac{\rho_{1} \times \operatorname{dot}\left(\vec{n}_{1},\left(v_{11}-s_{1}\right)\right)+\operatorname{dot}\left(\vec{n}_{1},\left(s_{1}-s_{2}\right)\right)}{\operatorname{dot}\left(\vec{n}_{1},\left(c_{2}-s_{2}\right)\right)}
\end{aligned}
$$

(iv) $(\mathrm{E}, \mathrm{V})$ contact as an instantaneous transition between (E,E) contact and (E',E') contact is the single scaling pair

$$
\begin{aligned}
& \rho_{1}=\frac{\operatorname{det}\left[V S^{\prime}\right] \times \operatorname{det}[A]-\operatorname{det}[V S] \times \operatorname{det}\left[A^{\prime}\right]}{\operatorname{det}[V V] \times \operatorname{det}\left[A^{\prime}\right]-\operatorname{det}\left[V V^{\prime}\right] \times \operatorname{det}[A]} \\
& \rho_{2}=\frac{\operatorname{det}\left[\left(v_{12}-v_{11}\right), s_{1}-s_{2}+\rho_{1}\left(v_{11}-s_{1}\right), v_{22}-v_{21}\right]}{\operatorname{det}\left[\left(v_{12}-v_{11}\right), v_{21}-s_{2}, v_{22}-v_{21}\right]}
\end{aligned}
$$

(v) $(\mathrm{E}, \mathrm{V})$ contact as an instantaneous transition between $(\mathrm{F}, \mathrm{V})$ contact and $\left(\mathrm{F}^{\prime}, \mathrm{V}^{\prime}\right)$ contact is the single scaling pair

$$
\begin{aligned}
& \rho_{1}=\frac{-\operatorname{dot}(n s s) \bullet \operatorname{dot}(n c s) '+\operatorname{dot}(n s s) \cdot \operatorname{dot}(n c s)}{\operatorname{dot}(n a s) \bullet \operatorname{dot}(n c s) '-\operatorname{dot}(n a s) \cdot \operatorname{dot}(n c s)} \\
& \rho_{2}=\frac{\rho_{1} \times \operatorname{dot}\left(\vec{n}_{1},\left(a_{1}-s_{1}\right)\right)+\operatorname{dot}\left(\vec{n}_{1},\left(s_{1}-s_{2}\right)\right)}{\operatorname{dot}\left(\vec{n}_{1},\left(c_{2}-s_{2}\right)\right)}
\end{aligned}
$$

